\title{
Communication
}

\section{A New Metric for the Space of Macroscopic Parameters of Grain Interfaces}

\section{A. MORAWIEC}

Analysis of datasets of macroscopic grain boundary parameters is based on grouping boundaries with similar parameters. Therefore, a measure of similarity or a distance between interfaces is needed. The paper introduces a new interface distance function. The function has properties allowing for accounting for equivalences among points in the macroscopic parameter space. Moreover, it has a simple interpretation: it minimizes the sum of angles of crystallite rotations needed to transform one interface into the other.

https://doi.org/10.1007/s11661-019-05361-3

(C) The Author(s) 2019

Crystallographic description of grain interfaces in polycrystalline materials is frequently based on macroscopic interface parameters. ${ }^{[1,2]}$ In the recent past, numerous sets of macroscopic grain boundary data have been collected and analyzed; see, e.g., References 3-9 and references therein. These analyses require identifying similar interfaces. For this purpose, a measure of interface similarity is needed. It is convenient to assess the similarity using a formally defined metric or a distance between interfaces. Although this is not always explicitly stated in reports on large interface datasets, the notion of a distance is fundamental for rigorous analysis of the data. The distance is linked to volume in the space of macroscopic parameters, and the volume in turn is necessary for estimating frequencies of occurrence of different boundary types. In particular, the interface distances are essential for determining the

A. MORAWIEC is with the Institute of Metallurgy and Materials Science, Polish Academy of Sciences, Reymonta 25, 30-059 Kraków, Poland. Contact e-mail: nmmorawi@cyf-kr.edu.pl

Manuscript submitted April 1, 2019.

Article published online July 16, 2019 frequencies based on the kernel density estimation. ${ }^{[8-10]}$ They are also crucial for interpolation of functions on the space of macroscopic parameters; see, e.g., Reference 11. A number of distance functions have been proposed in the past. ${ }^{[12-18]}$ This paper introduces a new metric with a simple geometric interpretation. The distance between two interfaces represents a magnitude of crystallite rotations needed for transforming one interface into the other.

The macroscopic parameters of an interface between two (centrosymmetric) crystals are given in the (Cartesian) reference frame of one of the crystals. They specify the misorientation of the crystal on the other side of the interface and the outward directed normal to the interface plane. In description of homophase interfaces, the cases with zero misorientation angles ("no-boundary singularity") are excluded from the macroscopic domain. With $M$ denoting a $3 \times 3$ special orthogonal matrix representing the misorientation and $n_{1}$ being a unit vector normal in the frame of the first crystal, the pair $\left(M, n_{1}\right)$ identifies macroscopically the interface from the viewpoint of the first grain. From the viewpoint of the second grain, this interface is represented by the pair $\left(M^{T}, n_{2}\right)$, where $n_{2}=-M^{T} n_{1} \cdot{ }^{[12]}$

By definition, a metric space is a set of points with a non-negative distance function $d$ such that $d(x, x)=0$, $d(x, y)=0$ implies $x=y$ (identity of indiscernibles), $d(x, y)=d(y, x) \quad$ (symmetry) and $d(x, y)+d(y, z) \geq$ $d(x, z)$ (triangle inequality) for every $x, y$ and $z .{ }^{[19]} \mathrm{A}$ distance function in the space of macroscopic parameters needs to satisfy some additional symmetry conditions. Generally, a physically unique interface has multiple symmetrically equivalent representations in the space of macroscopic parameters, and the distance between given two points in that space must be equal to the distance between their equivalents. The standard approach is to construct an underlying (asymmetric) distance function, and then to modify it to account for symmetries. ${ }^{[12]}$ One of the symmetries is applicable only to homophase interfaces. It is referred to as the grain exchange symmetry and corresponds to equivalence of $\left(M, n_{1}\right)$ and $\left(M^{T}, n_{2}\right)$. Other symmetries are mandatory and concern both homo- and hetero-phase interfaces. With the assumed centrosymmetry of crystals, inversion of the interface (plane and crystals) leaves the physical configuration unchanged, but changes the macroscopic parameters as the normal to the interface plane takes the opposite direction. This 'interface inversion' symmetry corresponds to equivalence of $\left(M, n_{1}\right)$ and $\left(M,-n_{1}\right)$. One also needs to account for the rotational crystal symmetries, i.e., the elements of the crystal point groups. They can be easily dealt with using $4 \times 4$ interface matrices ${ }^{[12,15]}$ of the type 


$$
B=\left[\begin{array}{cc}
0 & n_{2}^{T} \\
n_{1} & M
\end{array}\right] .
$$

Let $t_{i}(i=1,2)$ be a special orthogonal matrix representing a rotation of the $i$ th crystal, and let

$$
T_{i}=\left[\begin{array}{cc}
1 & 0^{T} \\
0 & t_{i}
\end{array}\right]
$$

With a given interface matrix $B$, also $T_{1} B T_{2}^{T}$ is an interface matrix. The transformation of $B$ to $T_{1} B T_{2}^{T}$ corresponds to a concomitant change of orientations of the first and second crystals by $T_{1}$ and $T_{2}$, respectively. With $T_{i}=C_{i}$ representing an element (proper rotation) of the point group of $i$ th crystal, the point $C_{1} B C_{2}^{T}$ is symmetrically equivalent to $B$.

It is easy to see that if $B$ represents the interface from the viewpoint of the first grain, then $B^{T}$ represents the same interface from the viewpoint of the second grain. The symbol $B^{-}$will denote the interface 'inverted' with respect to $B$, i.e., with $B$ corresponding to $\left(M, n_{1}\right), B^{-}$ corresponds to $\left(M,-n_{1}\right)$.

The focus below will be on two interfaces $x$ and $y$ represented by the pairs $\left(M_{x}, n_{x, 1}\right)$ and $\left(M_{y}, n_{y, 1}\right)$, or equivalently, by the corresponding matrices $B_{x}$ and $B_{y}$. For defining the new distance function, the following observation will be needed: For arbitrary interface matrices $B_{x}$ and $B_{y}$, there exist matrices $T_{1}$ and $T_{2}$ of the form [2] such that

$$
T_{1} B_{x} T_{2}^{T}=B_{y} ;
$$

$T_{1}$ and $T_{2}$ are not unique as Eq. [3] imposes five conditions on six independent parameters (three parameters per each rotation).

The definition of the new distance is based on minimization of combined magnitudes of the rotations $T_{i}$ transforming $B_{x}$ into $B_{y}$ via Eq. [3]. The minimized quantity is the sum of the angles $\omega_{i}$ of the rotations represented by $T_{i}$

$$
d\left(B_{x}, B_{y}\right)=\min \left(\omega_{1}+\omega_{2}\right) .
$$

One might consider minimizing $\omega_{1}+\omega_{2}$ over multiple parameters of $T_{i}$, but the result can be more simply obtained by minimization over just one continuous parameter and a discrete sign indicator. To demonstrate this, let the axis of the rotation represented by $t_{1}$ be along a unit vector $m$, and let the rotation angle satisfy the conditions $0 \leq \omega_{1} \leq \pi$. The matrix $t_{1}$ has the entries

$$
t_{1}^{i j}=\delta^{i j} \cos \omega_{1}+m^{i} m^{j}\left(1-\cos \omega_{1}\right)-\sum_{k} \varepsilon_{i j k} m^{k} \sin \omega_{1}
$$

where $m^{i}(i=1,2,3)$ are components of $m$. Equation [3] implies that

$$
t_{1} n_{x, 1}=n_{y, 1} .
$$

Thus, the vector $m$ is in the plane spanned by the vectors $n^{\|}=n_{x, 1}+n_{y, 1}$ and $n^{\perp}=n_{x, 1} \times n_{y, 1}$. The position of $m$ can be specified by an angle, say $\alpha(0 \leq \alpha \leq \pi)$, and a sign indicator $l= \pm 1$

$$
m=\hat{n}^{\|} l \sqrt{1-\cos ^{2} \alpha}+\hat{n}^{\perp} \cos \alpha,
$$

where the hat ${ }^{\wedge}$ denotes vector normalization. Substitution of $t_{1}$ in $\left(t_{1} n_{x, 1}\right) \cdot n_{y, 1}=n_{y, 1} \cdot n_{y, 1}=1$ by [5] leads to the dependence of $\cos \alpha$ on $\omega_{1}$

$$
\cos \alpha=\cot \left(\omega_{1} / 2\right) \tan (\beta / 2),
$$

where $\beta=\arccos \left(n_{x, 1} \cdot n_{y, 1}\right)$. For given $B_{x}$ and $B_{y}$, the matrices $T_{1}$ and $t_{1}$ satisfying Eq. [6] depend only on $\omega_{1}$ and $l$, and since $T_{2}=B_{y}^{-1} T_{1} B_{x}$, also the angle $\omega_{2}=\arccos \left(\operatorname{Tr}\left(\mathrm{T}_{2}\right) / 2-1\right)$ is a known function of $\omega_{1}$ and l, i.e., $\omega_{2}=\omega_{2}\left(\omega_{1}, l\right)$. Thus, the distance $d$ can be calculated by numerical minimization over continuous $\omega_{1}$ and discrete $l$

$$
d\left(B_{x}, B_{y}\right)=\min _{\omega_{1}, l}\left(\omega_{1}+\omega_{2}\left(\omega_{1}, \imath\right)\right),
$$

where $\beta \leq \omega_{1} \leq \pi$ and $l= \pm 1$.

With $m$ given by [7], there are singularities at $n_{x, 1}= \pm n_{y, 1}$. If $n_{x, 1}=+n_{y, 1}$, then $m=n_{x, 1}$, the minimum of $\omega_{1}+\omega_{2}\left(\omega_{1}, l\right)$ is at $\omega_{1}=0$, and the distance is

$$
\begin{aligned}
& d\left(B_{x}, B_{y}\right)=\arccos \left(\operatorname{Tr}\left(B_{y}^{-1} B_{x}\right) / 2-1\right) \\
& =\arccos \left(\left(\operatorname{Tr}\left(M_{y}^{T} M_{x}\right)-1\right) / 2\right) .
\end{aligned}
$$

In the case of $n_{x, 1}=-n_{y, 1}$, the angle $\omega_{1}$ is fixed at $\pi$ and $\omega_{2}$ is independent with the smallest value equal to the angle between the vectors $n_{x, 2}=-M_{x}^{T} n_{x, 1}$ and $n_{y, 2}=-M_{y}^{T} n_{y, 1}$, i.e.,

$$
d\left(B_{x}, B_{y}\right)=\pi+\arccos \left(n_{x, 2} \cdot n_{y, 2}\right)
$$

In practice, a simple way to overcome the problem with $n_{x, 1}= \pm n_{y, 1}$ is to use the property [16] given below.

Demonstrating that $d$ complies with the conditions for the distance function and the interface symmetry requirements is slightly tedious but straightforward. Clearly, $d\left(B_{x}, B_{x}\right)=0$ as the corresponding form of Eq. [3] is satisfied by $T_{1}=I=T_{2}$ ( $I$ is the $4 \times 4$ identity matrix) and both $\omega_{1}$ and $\omega_{2}$ take the smallest possible value of zero. By similar arguments, $d$ is shown to satisfy the identity of indiscernibles; the condition $d\left(B_{x}, B_{y}\right)=0$ implies that both $\omega_{1}$ and $\omega_{2}$ are zero, which means that both $T_{1}$ and $T_{2}$ are the identity matrices, and Eq. [3] leads to $B_{x}=B_{y}$. The symmetry $d\left(B_{x}, B_{y}\right)=d\left(B_{y}, B_{x}\right)$ follows directly from the facts that the angles of the mutually inverse rotations $T_{i}$ and $T_{i}^{T}$ are equal and that Eq. [3] implies $B_{x}=T_{1}^{T} B_{y} T_{2}$. To 
show the triangle inequality, one needs to recall that the angle of rotation constitutes a distance in the space of rotations. Let the distance function be called $\varpi$. For a rotation $X$ by the angle $\omega_{X}$, one has $\omega_{X}=\varpi(X, I)$. Moreover, $\varpi(X Y, I)=\varpi\left(X, Y^{T}\right)$ for arbitrary rotations $X$ and $Y$. To a given pair $B_{x}, B_{y}$ corresponds a pair of rotations $T_{1}^{y x}, T_{2}^{y x}$ satisfying Eq. [3] and such that their rotation angles $\omega_{1}$ and $\omega_{2}$ minimize the sum $\omega_{1}+\omega_{2}$. There are also analogous matrices $T_{i}^{z y}$ and $T_{i}^{z x}$ corresponding to the pairs $B_{y}, B_{z}$ and $B_{x}, B_{z}$, respectively. One can write

$$
\begin{aligned}
& d\left(B_{x}, B_{y}\right)+d\left(B_{y}, B_{z}\right)=\varpi\left(T_{1}^{y x}, I\right)+\varpi\left(T_{2}^{y x}, I\right) \\
& \quad+\varpi\left(T_{1}^{z y}, I\right)+\varpi\left(T_{2}^{z y}, I\right) .
\end{aligned}
$$

As the angle of a rotation is equal to the angle of its inverse, the above sum can be written in the form $\varpi\left(T_{1}^{y x}, I\right)+\varpi\left(\left(T_{1}^{z y}\right)^{T}, I\right)+\varpi\left(T_{2}^{y x}, I\right)+\varpi\left(\left(T_{2}^{z y}\right)^{T}, I\right)$, and since $\varpi$ satisfies the triangle inequality, this quantity is not smaller than

$$
\begin{gathered}
\varpi\left(T_{1}^{y x},\left(T_{1}^{z y}\right)^{T}\right)+\varpi\left(T_{2}^{y x},\left(T_{2}^{z y}\right)^{T}\right) \\
\quad=\varpi\left(T_{1}^{z y} T_{1}^{y x}, I\right)+\varpi\left(T_{2}^{z y} T_{2}^{y x}, I\right) .
\end{gathered}
$$

Now, since $T_{1}^{z y} T_{1}^{y x} B_{x}\left(T_{2}^{z y} T_{2}^{y x}\right)^{T}=B_{z}=T_{1}^{z x} B_{x}\left(T_{2}^{z x}\right)^{T}$, the above sum is not smaller than the minimal

$$
\varpi\left(T_{1}^{z x}, I\right)+\varpi\left(T_{2}^{z x}, I\right)=d\left(B_{x}, B_{z}\right) .
$$

Hence, $d\left(B_{x}, B_{y}\right)+d\left(B_{y}, B_{z}\right)$ is not smaller than $d\left(B_{x}, B_{z}\right)$. This shows that $d$ is a distance function.

The distance function $d$ is invariant with respect to symmetry operations on the space of macroscopic parameters. First, if $T_{1}$ and $T_{2}$ transform $B_{x}$ to $B_{y}$ as in Eq. [3], the same matrices also transform $B_{x}^{-}$to $B_{y}^{-}$, i.e., $T_{1} B_{x}^{-} T_{2}^{T}=B_{y}^{-}$. Hence, the minimum of the sum of the angles $\omega_{i}$ is in both cases the same and

$$
d\left(B_{x}^{-}, B_{y}^{-}\right)=d\left(B_{x}, B_{y}\right),
$$

i.e., the 'interface inversion' is an isometry with respect to $d$. Second, if $T_{1}$ and $T_{2}$ transform $B_{x}$ to $B_{y}$ as in Eq. [3], the matrices $T_{2}$ and $T_{1}$ transform $B_{x}^{T}$ to $B_{y}^{T}$, i.e., $T_{2} B_{x}^{T} T_{1}^{T}=B_{y}^{T}$. Hence,

$$
d\left(B_{x}^{T}, B_{y}^{T}\right)=d\left(B_{x}, B_{y}\right),
$$

i.e., the grain exchange is an isometry with respect to $d$. Third, if $T_{1}$ and $T_{2}$ transform $B_{x}$ to $B_{y}$ as in Eq. [3], the matrices $C_{1} T_{1} C_{1}^{T}$ and $C_{2} T_{2} C_{2}^{T}$ transform $C_{1} B_{x} C_{2}^{T}$ to $C_{1} B_{y} C_{2}^{T}$. The angle of the rotation $C_{i} T_{i} C_{i}^{T}$ is equal to that of the rotation $T_{i}$. Hence, one has

$$
d\left(C_{1} B_{x} C_{2}^{T}, C_{1} B_{y} C_{2}^{T}\right)=d\left(B_{x}, B_{y}\right),
$$

i.e., the symmetry transformation $B \rightarrow C_{1} B C_{2}^{T}$ is an isometry with respect to $d$.

With the above described underlying distance function $d$, the proper distance accounting for the interface symmetries has the form
Table I. The Distances $\chi, d_{\text {Angle }}, \Delta_{12}, \Omega_{A B, C D}$ and $d$ between example interfaces of Ref. [16]

\begin{tabular}{lll}
\hline & Ex. 1 & Ex. 2 \\
\hline$\chi$ & $\sqrt{5} \delta_{1}$ & $\delta_{2}$ \\
$d_{\text {Angle }}$ & $2 \delta_{1}$ & $2 \delta_{2}$ \\
$\Delta_{12}$ & $2 \delta_{1}$ & $\delta_{3}$ \\
$\Omega_{A B, C D}$ & $\delta_{1}$ & $\delta_{2}$ \\
$d$ & $2 \delta_{1}$ & $2 \delta_{2}$ \\
\hline
\end{tabular}

The quantities $\delta_{i}(i=1,2,3) \quad$ are $\quad \delta_{i}=\arccos \left(u_{i}\right) / 2$, where $u_{1}=56 / 65, u_{2}=33 / 63$, and $u_{3}=37 / 63$.

$$
\tilde{d}\left(B_{x}, B_{y}\right)=\min _{i} d\left(B_{x}, S_{i}\left(B_{y}\right)\right),
$$

where $S_{i}$ is the 'interface inversion' symmetry or one of the rotational symmetries $\left(C_{1} B C_{2}^{T}\right)$ or (if applicable) the grain exchange symmetry or a composition of these symmetries. ${ }^{[15]}$

The distance function $d$ is naturally linked to orientations of crystallites and has a simple interpretation: if one of the crystals forming an interface is rotated, the new configurations deviates from original one by the angle of the rotation, i.e., with $T$ representing an arbitrary rotation by $\omega$, one has $d(B, T B)=d\left(B, B T^{T}\right)=\omega$.

The function $d$ is similar to the metric $d_{\text {Angle }}$ of Olmsted, ${ }^{[14]}$ but differently than in the case of Olmsted's distance, there is no need use coordinates such that the boundary plane is given by $z=0$, and there is no ambiguity in macroscopic interface representation (embodied in the Olmsted's $U$ matrix $\left.{ }^{[14]}\right)$. It is also worth noting that in general the $p$-norm-based functions $\min \left(\omega_{1}^{p}+\omega_{2}^{p}\right)^{1 / p}(p>1)$ also satisfy the conditions for the underlying interface distance.

To give explicit examples, distances for two special pairs of interfaces listed in Reference 16 are calculated and juxtaposed with results of other methods. In the first example (Ex. 1), the misorientations $M_{x}$ and $M_{y}$ correspond to rotations about $\left[\begin{array}{lll}0 & 1 & 0\end{array}\right]^{T}$ by the angles $2 \arctan (1 / 5)$ and $2 \arctan (1 / 2)$, respectively, $n_{x, 1} \propto\left[\begin{array}{lll}1 & 0 & 5\end{array}\right]^{T}$ and $n_{y, 1} \propto\left[\begin{array}{lll}1 & 0 & 2\end{array}\right]^{T}$. In the second example (Ex. 2), $M_{x}=M_{y}$ is the rotation about $\left[\begin{array}{lll}1 & 1 & 0\end{array}\right]^{T}$ by $\arctan (1 / 3), \quad n_{x, 1} \propto\left[\begin{array}{lll}3 & 1 & 2\end{array}\right]^{T} \quad$ and $\quad n_{y, 1} \propto\left[\begin{array}{lll}7 & -1 & 2\end{array}\right]^{T}$. Table I lists the values of the distance $d$ between $\left(M_{x}, n_{x, 1}\right)$ and $\left(M_{y}, n_{y, 1}\right)$ along the distances $\chi$ of Reference 12, Eq. 3, $d_{\text {Angle }}$ of Reference 14, $\Delta_{12}$ of Reference 16, Eq. 4.3, $\Omega_{A B, C D}$ of Reference 18, Eq. 22. For a more complete comparison of all known interface distance functions, the reader is referred to the forthcoming article. ${ }^{[20]}$ The article also describes an alternative (analytical) method for calculating the distance $d$.

\section{OPEN ACCESS}

This article is distributed under the terms of the Creative Commons Attribution 4.0 International License (http://creativecommons.org/licenses/by/4.0/), which permits unrestricted use, distribution, and re- 
production in any medium, provided you give appropriate credit to the original author(s) and the source, provide a link to the Creative Commons license, and indicate if changes were made.

\section{REFERENCES}

1. C. Goux: Mém. scient. Rev. Métall., 1961, vol. 58, pp. 661-76.

2. A. Sutton and R. Ballufi: Interfaces in Crystalline Materials, Clarendon Press, Oxford, 1995.

3. D. Saylor, A. Morawiec, and G. Rohrer: Acta Mater., 2003, vol. 51, pp. 3663-74.

4. G. Rohrer: J. Am. Ceram. Soc., 2011, vol. 94, pp. 633-46.

5. F. Zhang and D. Field: Metall. Mater. Trans. A, 2013, vol. 44A, pp. 4927-36.

6. E. Farabi, P. Hodgson, G. Rohrer, and H. Beladi: Acta Mater., 2018, vol. 154, pp. 147-60.

7. N. Haghdadi, P. Cizek, P.D. Hodgson, V. Tari, G.S. Rohrer, and H. Beladi: Philos. Mag., 2018, vol. 98, pp. 1284-306.

8. H. Pirgazi, K. Glowinski, A. Morawiec, and L. Kestens: J. Appl. Crystallogr., 2015, vol. 48, pp. 1672-8.
9. M. Kelly, K Glowinski, N Nuhfer, and G Rohrer: Acta Mater., 2016, vol. 111, pp. 22-30.

10. K. Glowinski and A. Morawiec: Metall. Mater. Trans. A, 2014, vol. 45A, pp. 3189-94.

11. V. Bulatov, B. Reed, and M. Kumar: Acta Mater., 2014, vol. 65, pp. 161-75.

12. A. Morawiec: Grain Growth in Polycrystalline Materials III, B. Adams, A. Rollett, and H. Weiland, eds., TMS, Warrendale, PA, USA, 1998, pp. 509-14.

13. J. Cahn and J. Taylor: J. Mater. Sci., 2006, vol. 41, pp. 7669-74.

14. D. Olmsted: Acta Mater., 2009, vol. 57, pp. 2793-99.

15. A. Morawiec: J. Appl. Crystallogr., 2009, vol. 42, pp. 783-92.

16. A. Sutton, E. Banks, and A. Warwick: Proc. R. Soc. A, 2015, vol. 471A, p. 20150442.

17. A. Morawiec: Proc. R. Soc. A, 2016, vol. 472A, p. 20150741.

18. T. Francis, I. Chesser, S. Singh, E. Holm, and M. De Graef: Acta Mater., 2019, vol. 166, pp. 135-47.

19. M. Deza and E. Deza: Encyclopedia of Distances, 4th ed., Springer, Berlin, 2016.

20. A. Morawiec: Unpublished Research, 2019.

Publisher's Note Springer Nature remains neutral with regard to jurisdictional claims in published maps and institutional affiliations. 